\title{
Defining Radicals: Comparison Between Language Style Used in Social Media Between Government-Approved and Radical Moslem Group
}

\author{
Rian Hardinata ${ }^{1}$, Liestyowati ${ }^{2}$ \\ ${ }^{1}$ Telecommunication Department, Akademi Telkom Sandhy Putra Jakarta, Jakarta, Indonesia \\ ${ }^{2}$ Telecommunication Department, Akademi Telkom Sandhy Putra Jakarta, Jakarta, Indonesia \\ rianhardinata@akademitelkom.ac.id (Rian Hardinata), liestyowati@akademitelkom.ac.id (Liestyowati)
}

\begin{abstract}
Lately, there is a rise on Muslim radical groups topic in Indonesia. Hizbut Tahrir Indonesia (HTI) is one of a group deemed as the militant group that endangers Indonesia's unity according to the official statement by Wiranto, Minister for Political, Legal, and Security Affairs, on behalf of Indonesian government position towards the HTI status. Albeit their radical nature, their lives are quite modern in the opposite. It is proven by the usage of social media like Twitter and Facebook as their means of communication. Twitter is one of the most efficient methods of communication to this religious group followers, as well as other Islamic religious groups such as MUI and NU. This research is intended to find the differences of content regarding language style used by HTI and those government approved Muslim groups in Indonesia (MUI and NU). The tweets were extracted, then compared based on commonly used words and phrases. The purpose of this approach is to know the difference between government-approved religious groups' tweets and the tweets of the so-called radical group by the government. It has found that there are some differences in language style used by each Muslim group which could be used to detect similar militant groups in the future.
\end{abstract}

Keywords Data Mining, Text Mining, Sentiment Analysis

\section{Introduction}

The growth of religion based radical organisations is dangerous to a country. Not only it creates a disturbance in harmonious nature inside it, but also have a potential to spread chaos around a region. Therefore defining what is radical and what is not are important.

Currently, there is no set of rule from Indonesian government that determine what activity that is considered radical in social media, in addition to difficulties on supervising activity from government caused by the difference of geographical condition in Indonesia resulted in flourishment of religion based radical group.

Social media is one of the most effective media to use by these groups to spread their belief, however, currently, there is no initiative of the Indonesian government to supervise this popular channel. Therefore, it is important to define radical activity on social media. This research aims to search a method that could serve as early warning of radical group activities in social media.

Sentiment analysis is a method commonly used to identify nature of a text, the most common practice of sentiment analysis is detecting if a text belongs to a class of sentiment, usually based on a collection of terms inside of the document that was defined before. For example in previous research by Rian Hardinata, similar method is used ti identify whether a tweet is a spam or not based on a list of word that has been put in a class before.

As has been described before currently there are no rules from the government of Indonesia regarding radical social media content; therefore this research will try to fill the gap by providing a method to detect radical activity group based on the sentiment of the content that they post on social media.

It has been known before that the radical group in social media utilise offensive message to persuade their follower or just to agitate everybody whose not on the same way thinking as them[1]. Most of the words they use usually should be moving toward negative sentiment, therefore, we hypothesise that radical group in social media uses more negative sentiment words than the government-approved group.

3200 Tweets extracted from an account of government-approved Islamic group (Nahdatul Ulama/NU) and Islamic group that is deemed radical by the government (Hizbut Tahrir Indonesia/HTI) using Python. Then all the tweets processed and converted into a bag of words, after that the phrase is being analysed using a list of negative and positive opinion words. The research finds that radical group account uses the more negative word rather than government-approved Muslim group.

Our method could be utilised as an early warning of a possible radical group before its even start to become 
significant by analysing its social media activity. Thus it will be easier to handle by the government rather than handling radical group that has been matured before.

\section{Research Methodology}

The methodology used in this research consists of several main steps which is often, tweet gathering, tweet cleaning, converting tweet to the bag of words and word sentiment analysis.

\subsection{Getting Twitter API Keys}

Twitter open the API to ease the process of data gathering, in order to access this API, registration process is needed for a developer account[2]. After this process, a person who registers the account will get some secret keys that will be used to gather Tweet via Twitter API using their tools of choice.

\subsection{Data Gathering Via API and Python}

The keys that are obtained from the previous step is inserted into tools to gather data from Twitter. This research uses Open source Python application called Tweepy[3], Tweepy is a package that is installed and controlled via python.

The output of this package is CSV file which contains tweets, date of the tweets, tweet text, tweets retweet and tweets favourite count.

\subsection{Tweet Data Profile}

To comply with Twitter rules, the data that we could obtain through tweepy is limited around 3200 latest tweets only[4].

For This research we use two moslem religious group to gather tweet data, to represent a religious group that is approved by the government, we use data from official Nahdatul Ulama (NU) account (@nu_online).

Meanwhile to represent a religious group that is not approved by the government or in another word, deemed radical by the government, is Hizbut Tahrir Indonesia (@ hizbuttahririd). Both Twitter accounts are chosen because it is claimed as official by respective organisation.

\subsection{Tweet Cleaning}

Before it could be used regularly, Tweet gathered from the previous step should be cleaned. Cleaning process has a purpose to clean data from the noise that might included at the time tweet collected from the previous process.

In this cleaning process, we remove stop words, to remove stop words we used a list from the research of Tala.[5]. We also remove white spaces and URL, it is deleted because we see that there are many URL shortener usages inside tweet data (e.g. www.example.com or http://www.ex.am), and we also change the case to lower case to make it easier to detect using our sentiment word library.

In this research every tweet cleaning process used $R$ software, $\mathrm{R}$ is originally statistic software but have some packages that usually used on Natural Language Processing, in this research we use several packages for R, mainly we use a package called $\mathrm{tm}[6]$.

\subsection{Converting Tweet to Bag of Word}

It is hard to analyse 3200 tweets one by one individually; therefore tweet data obtained from the Twitter should be simplified, one of the method to simplify document is by changing it into a bag of words.

Because we want to represent tweet as a whole, we used unigram as a choice; unigram is a unique case of n-gram where $n=1[7]$, thus a connection between words is not recorded.

After cleaning process, 3200 tweets converted into the bag of words using R software[8], this process makes it easy to conclude overall sentiment of each moslem group Twitter account.

\subsection{Indonesian Negative Word Sentiment Dictionary}

To do sentiment analysis, data of words that tagged with its sentiment is necessary, in this research we use negative sentiment dictionary from Wahid [9] Research.

This word originated from Liu [10] study, which is modified or translated to the Indonesian language.

\subsection{Word Sentiment Analysis}

Every word in our bag of words checked against Wahid's sentiment dictionary. If the word is a negative word that is available in Wahid's dictionary, then negative word count of that document is increasing.

\section{Result}

By doing procedures in research method, we retrieve several data, from 3200 tweets, after cleaning, we found that from NU Twitter account there are 4309 words; meanwhile, 6835 words were extracted from HTI Twitter account.

By using the negative word from Indonesian negative word dictionary, we found that from 4309 of the individual words, NU tweets contains about 246 negative words $(5,7 \%)$. Meanwhile using the same procedure, from 6835 different words, HTI tweets contains about 450 negative words $(6,5 \%)$.

From 17385 total words that are used to create their tweet, NU only use 752 words $(4,3 \%)$ with negative sentiment; meanwhile, HTI used 1623 words $(6,4 \%)$ out of 24972 total words with negative sentiment.

Table 1. Top 10 Negative Words On NU and HTI Twitter Account

\begin{tabular}{|c|c|c|}
\hline \multirow{2}{*}{ No. } & \multicolumn{2}{|c|}{ Twitter Account } \\
\cline { 2 - 3 } & NU & HTI \\
\hline 1 & Neraka & Asing \\
\hline 2 & Mati & Terorisme \\
\hline 3 & Bertemu & Kafir \\
\hline 4 & Murah & Ancaman \\
\hline 5 & Pagar & Kekerasan \\
\hline 6 & Salah & Perjuangan \\
\hline 7 & Sakit & Salah \\
\hline 8 & Alasan & Teror \\
\hline 9 & Peringatan & Perubahan \\
\hline 10 & Maaf & Kejahatan \\
\hline
\end{tabular}


Several negative sentiment words have a high frequency of appearance. It described in Table 1.

To understand Furthermore, we gather tweet based on top 3 words on table 1 per twitter account. From NU account, the three keywords are "Neraka", "Mati" and "bertemu". Using Keyword "Neraka", from our data we found that there is 21 tweet that uses that word, top 10 most retweeted tweets could be described on the table below.

Table 2. Top $10 \mathrm{NU}$ Tweets Using "Neraka" Keyword

\begin{tabular}{|c|c|c|}
\hline \multirow{2}{*}{ No. } & \multicolumn{2}{|l|}{ Tweet Details } \\
\hline & Tweet Content & Retweet \\
\hline 1 & $\begin{array}{l}\text { â€œPuasa itu perisai yang dipergunakan seorang } \\
\text { hamba untuk membentengi dirinya dari siksaan } \\
\text { neraka.â€ } \square \text { (Hadist } \\
\text { https://t.co/f2RoMiBX90 }\end{array}$ & 58 \\
\hline 2 & $\begin{array}{l}\text { Kisah Orang Tekun Ibadah yang Masuk Neraka } \\
\text { https://t.co/aOTcEJPsWh } \\
\text { https://t.co/QAaiAND9yQ }\end{array}$ & 42 \\
\hline 3 & $\begin{array}{l}\text { Masuk Neraka Gara-gara Air Wudhu? } \\
\text { https://t.co/vS9MXc5E9P https://t.co/9S9xueZdzt }\end{array}$ & 36 \\
\hline 4 & $\begin{array}{l}\text { Kisah Budak Batal Masuk Neraka Karena Sedek- } \\
\text { ah } \\
\text { https://t.co/0XHQFle9Hk https://t.co/3rsg6ETvRN }\end{array}$ & 17 \\
\hline 5 & $\begin{array}{l}\text { Kisah Orang Tekun Ibadah yang Masuk Neraka } \\
\text { https://t.co/aOTcEK73NP } \\
\text { https://t.co/dhNXT8eUkk }\end{array}$ & 17 \\
\hline 6 & $\begin{array}{l}\text { Kisah Budak Batal Masuk Neraka Karena Sedek- } \\
\text { ah } \\
\text { https://t.co/0XHQFle9Hk } \\
\text { https://t.co/9NVXOwbV6T }\end{array}$ & 14 \\
\hline 7 & $\begin{array}{l}\text { Benarkah Orang Tua Nabi Muhammad SAW } \\
\text { Penghuni Neraka? } \\
\text { https://t.co/Bil78iRaYk https://t.co/pbCZvdOdAn }\end{array}$ & 14 \\
\hline 8 & $\begin{array}{l}\text { Masuk Neraka Gara-gara Air Wudhu? } \\
\text { https://t.co/vS9MXc5E9P } \\
\text { https://t.co/Lzh1EYZgT1 }\end{array}$ & 14 \\
\hline 9 & $\begin{array}{l}\text { Kisah Budak Batal Masuk Neraka Karena Sedek- } \\
\text { ah } \\
\text { https://t.co/0XHQFkWyiK } \\
\text { https://t.co/gNgTN3nlOp }\end{array}$ & 14 \\
\hline 10 & $\begin{array}{l}\text { Dua Kisah Calon Ahli Neraka yang Masuk Surga } \\
\text { https://t.co/lvSjWhJ4yq https://t.co/sdkpEkBftM }\end{array}$ & 13 \\
\hline
\end{tabular}

Meanwhile using keyword "Mati", there are 43 tweets which use this word, ten most retweeted tweets described in the table below.

Table 3. Top 10 NU Tweets Using "Mati” Keyword

\begin{tabular}{|c|c|c|}
\hline \multirow{2}{*}{ No. } & \multicolumn{2}{|l|}{ Tweet Details } \\
\hline & Tweet Content & Retweet \\
\hline 1 & $\begin{array}{l}\text { Saya Indonesia! Saya Pancasila! NKRI Harga } \\
\text { Mati... https://t.co/CFsI7Pz9Io }\end{array}$ & 248 \\
\hline 2 & $\begin{array}{l}\text { Semua perempuan adalah kaum ibumu maka } \\
\text { hormatilah dan jangan pernah melecehkannya.[11] } \\
\text { \#GusMus https://t.co/yqf0L1Ezuv }\end{array}$ & 121 \\
\hline 3 & $\begin{array}{l}\text { Konsep NKRI itu sudah final } \\
\text { \#hargamati yang tidak bisa ditawar lagi. \#Habib- } \\
\text { Luthfi https://t.co/iNUWhgAOzL }\end{array}$ & 50 \\
\hline 4 & $\begin{array}{l}\text { Ketika KH Hasyim Asyâ€ } €^{\mathrm{TM}} \text { ari Turunkan Bedug } \\
\text { untuk Hormati Tamu } \\
\text { https://t.co/VWfwsrAWS4 } \\
\text { https://t.co/EQUncrqFJm }\end{array}$ & 47 \\
\hline 5 & $\begin{array}{l}\text { Habib Syech: Pesan Mbah Idris, Nikmatilah Hidup } \\
\text { Meskipun Susah } \\
\text { https://t.co/SELaBC40ED } \\
\text { https://t.co/3qzMjmgB8M }\end{array}$ & 43 \\
\hline
\end{tabular}

Table 3, cont.

\begin{tabular}{|c|l|c|}
\hline 6 & $\begin{array}{l}\text { Mbah Liem Pencetus â€ }{ }^{\sim} \text { NKRI Harga Mati, Pan- } \\
\text { casila Jayâ̂€TM } \\
\text { https://t.co/kL9uwWNy5T } \\
\text { https://t.co/ahKGTGoArZ }\end{array}$ & 35 \\
\hline 7 & $\begin{array}{l}\text { Hadiah Orang Hidup Kepada Orang Mati } \\
\text { https://t.co/viJKptmJxB https://t.co/gEaXJOZ0Lc }\end{array}$ & 33 \\
\hline 8 & $\begin{array}{l}\text { Tiga Tokoh yang Dihormati Gus Dur } \\
\text { https://t.co/qMbdjNfnxK https://t.co/zJ8ssRG4jM }\end{array}$ & 32 \\
\hline 9 & $\begin{array}{l}\text { Kisah Unik Gus Dur Menulis Pengatar Buku } \\
\text { â€œMati Ketawa Cara Rusiaâ€• } \\
\text { https://t.co/QJOeVKQPkk } \\
\text { https://t.co/anpmDoTGWE }\end{array}$ & 29 \\
\hline 10 & $\begin{array}{l}\text { NKRI Harga Mati, NU Bukan Latah dan Tiru-tiru } \\
\text { https://t.co/LMyRekihtZ } \\
\text { https://t.co/wXwzX3MLAs }\end{array}$ & 28 \\
\hline
\end{tabular}

Lastly, Using word "Bertemu", we found 18 tweets on NU twitter account, the tweet described on the table below.

Table 4. Top $10 \mathrm{NU}$ Tweets Using "Bertemu" Keyword

\begin{tabular}{|c|c|c|}
\hline \multirow{2}{*}{ No. } & \multicolumn{2}{|l|}{ Tweet Details } \\
\hline & Tweet Content & Retweet \\
\hline 1 & 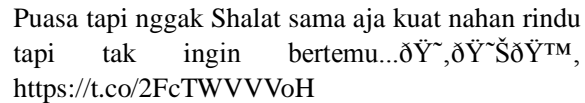 & 87 \\
\hline 2 & $\begin{array}{l}\text { Kisah Syekh Abdul Qadir Al-Jilani Mimpi Ber- } \\
\text { temu Nabi[12] } \\
\text { https://t.co/j0sN3pXV3k https://t.co/r0Hcyce04w }\end{array}$ & 27 \\
\hline 3 & $\begin{array}{l}\text { Kisah Syekh Abdul Qadir Al-Jilani Mimpi Ber- } \\
\text { temu Nabi[12] } \\
\text { https://t.co/j0sN3pGjEK } \\
\text { https://t.co/QVv18vYENo }\end{array}$ & 26 \\
\hline 4 & $\begin{array}{l}\text { Keistimewaan Orang Berpuasa, Bertemu Lang- } \\
\text { sung dengan Allah } \\
\text { https://t.co/vtY6Yrl8B5 https://t.co/u2SWzZZjqU }\end{array}$ & 22 \\
\hline 5 & $\begin{array}{l}\text { Kisah Syekh Abdul Qadir Al-Jilani Mimpi Ber- } \\
\text { temu Nabi[12] } \\
\text { https://t.co/j0sN3pGjEK https://t.co/gvkjbbSVbN }\end{array}$ & 22 \\
\hline 6 & $\begin{array}{l}\text { Kisah Syekh Abdul Qadir Al-Jilani Mimpi Ber- } \\
\text { temu Nabi[12] } \\
\text { https://t.co/j0sN3pGjEK } \\
\text { https://t.co/vEWDJkQOK9 }\end{array}$ & 20 \\
\hline 7 & $\begin{array}{l}\text { Mimpi Mbah Baedhowi Bertemu Rasulullah untuk } \\
\text { Mendirikan Cabang NU } \\
\text { https://t.co/vOCnc9WBdl } \\
\text { https://t.co/ZKcKYOgOyt }\end{array}$ & 17 \\
\hline 8 & $\begin{array}{l}\text { Kisah Syekh Abdul Qadir Al-Jilani Mimpi Ber- } \\
\text { temu Nabi[12] } \\
\text { https://t.co/j0sN3pXV3k https://t.co/THPR4qoeig }\end{array}$ & 17 \\
\hline 9 & $\begin{array}{l}\text { Kisah Syekh Abdul Qadir Al-Jilani Mimpi Ber- } \\
\text { temu Nabi[12] } \\
\text { https://t.co/j0sN3pGjEK https://t.co/4ZwRvHh6Bc }\end{array}$ & 15 \\
\hline 10 & $\begin{array}{l}\text { Kisah Syekh Abdul Qadir Al-Jilani Mimpi Ber- } \\
\text { temu Nabi[12] } \\
\text { https://t.co/j0sN3pXV3k } \\
\text { https://t.co/td8vfOMQmC }\end{array}$ & 14 \\
\hline
\end{tabular}


From HTI account with keyword "Asing", there are 43 using this word, ten most retweeted tweets described in the table below.

Table 5. Top 10 HTI Tweets Using “Asing” Keyword

\begin{tabular}{|c|c|c|}
\hline \multirow{2}{*}{ No. } & \multicolumn{2}{|l|}{ Tweet Details } \\
\hline & Tweet Content & Retweet \\
\hline 1 & $\begin{array}{l}\text { Wacanakan Asing Kelola Pulau, Luhut Dianggap } \\
\text { Pelaku Makar Sesungguhnya.[13] Baca } \\
\text { https://t.co/9G1Vfl0xpI https://t.co/qyzspeHJlz }\end{array}$ & 100 \\
\hline 2 & $\begin{array}{l}\text { Faham Islam Moderat, Ide Asing \&amp; Jauh dari } \\
\text { Rahmatan lil Alamin https://t.co/AjNY1yAKLD }\end{array}$ & 36 \\
\hline 3 & $\begin{array}{l}\text { Bahkan disinyalir terkait ttg isu kepemilikan } \\
\text { properti asing bisa jadi akal-akalan aseng utk } \\
\text { kuasai Indonesia \#Reklamasi4China }\end{array}$ & 26 \\
\hline 4 & $\begin{array}{l}\text { Kita perlu melihat bahwa \#Reklamasi4China } \\
\text { hanya menguntungkan kapitalis dan asing. Perlu } \\
\text { diinvestigasi kepentingan modal asing dsana. }\end{array}$ & 25 \\
\hline 5 & $\begin{array}{l}\text { Sudahi Penjajahan Asing di Indonesia } \\
\text { https://t.co/99FTRKQSoC }\end{array}$ & 23 \\
\hline 6 & $\begin{array}{l}\text { Penyerahan SDA kepada Asing Lebih Berbahaya } \\
\text { daripada Korupsi https://t.co/k28D19BkFO }\end{array}$ & 19 \\
\hline 7 & $\begin{array}{l}500 \text { Investor Asing Ngemplang Pajak, Ini Mem- } \\
\text { buktikan Bahwa[14]â } \epsilon_{\mid}^{\prime} \text { https://t.co/jdXGyTHX4D }\end{array}$ & 16 \\
\hline 8 & $\begin{array}{l}\text { 10.Negeri ini pun masih sangat bergantung pada } \\
\text { pihak asing dalam berbagai aspeknya. Tak hny } \\
\text { perusahaan } 2 \text { asing yg mencengkeram negeri ini. }\end{array}$ & 15 \\
\hline 9 & $\begin{array}{l}\text { 19. Di sisi lain, sekularisasi ini menerima ke- } \\
\text { percayaan-kepercayaan asing dan keyakinan lain } \\
\text { di bawah kedok â€œkeragaman budayaâ€• }\end{array}$ & 14 \\
\hline 10 & 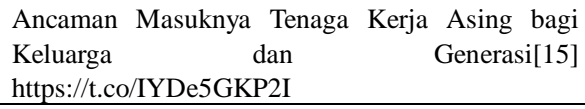 & 14 \\
\hline
\end{tabular}

Meanwhile using keyword "Terorisme", there are 45 tweets that used this word, 10 most retweeted tweets described in table below.

Table 6. Top 10 HTI Tweets Using "Terorisme" Keyword

\begin{tabular}{|c|c|c|}
\hline \multirow{2}{*}{ No. } & \multicolumn{2}{|l|}{ Tweet Details } \\
\hline & Tweet Content & Retweet \\
\hline 1 & $\begin{array}{lr}\text { Inilah Alasannya, Mengapa UU } & \text { Terorisme dan } \\
\text { Revisinya } & \text { Membidik } \\
\text { https://t.co/OPj69ERbbK } & \text { Islam } \\
\end{array}$ & 45 \\
\hline 2 & $\begin{array}{l}\text { Pembunuhan Siyono dan } \\
\text { https://t.co/EUvl2Vs6HR }\end{array}$ & 34 \\
\hline 3 & $\begin{array}{l}\text { Draf RUU Terorisme, Memproduksi dan Mele- } \\
\text { galkan Kemungkaran https://t.co/bItVRkKlxj }\end{array}$ & 32 \\
\hline 4 & $\begin{array}{l}\text { Menyoal Densus } 88 \text { dan Revisi UU Terorisme } \\
\text { https://t.co/4sIjQUuS4a }\end{array}$ & 28 \\
\hline 5 & $\begin{array}{l}\text { Membaca Drama Pelegalan } \\
\text { https://t.co/zFKNiilHIX }\end{array}$ & 25 \\
\hline 6 & $\begin{array}{l}\text { Audiensi HTI dan Ormas dengan Ketua Pansus } \\
\text { RUU Terorisme https://t.co/1rhZbG06cF }\end{array}$ & 18 \\
\hline 7 & $\begin{array}{l}\text { Talkshow Interaktif HTI Bandar Lampung: } \\
\text { â€œRevisi UU Terorisme Membidik Is- } \\
\text { lam?[16]â€• https://t.co/oQzNfs29fV }\end{array}$ & 18 \\
\hline 8 & $\begin{array}{l}\text { Ketua Pansus RUU Terorisme : Yang Tidak Se- } \\
\text { jalan Dengan Barat Dicap Teroris[17] } \\
\text { https://t.co/YhSVQWCY4N }\end{array}$ & 17 \\
\hline 9 & $\begin{array}{l}\text { Kritisi Revisi UU Terorisme, MUI DIY Undang } \\
\text { HTI Paparkan Sikapnya https://t.co/M6oVIeBsM7 }\end{array}$ & 17 \\
\hline 10 & $\begin{array}{l}\text { Kitab Hizbut Tahrir, Kitab Terorisme ? } \\
\text { https://t.co/IMcybeKWLb }\end{array}$ & 15 \\
\hline
\end{tabular}

And for the last, using keyword "Kafir", we found 45 tweets that used this word, ten most retweeted tweets described in the table below.

Table 7. Top 10 HTI Tweets Using "Kafir" Keyword

\begin{tabular}{|c|c|c|}
\hline \multirow{2}{*}{ No. } & \multicolumn{2}{|l|}{ Tweet Details } \\
\hline & Tweet Content & Retweet \\
\hline 1 & $\begin{array}{l}\text { \#StopKriminalisasiUlama \#AksiUmatPeduliJa- } \\
\text { karta } \\
\text { \#HaramPemimpinKafir } \\
\text { Pagi ini di kawasan Patung Kuda Jakarta. } \\
\text { https://t.co/AU3JkY5yLx }\end{array}$ & 305 \\
\hline 2 & $\begin{array}{l}\text { 4. Melarang memberi jalan bagi orang/negara kafir } \\
\text { menguasai umat Islam [18] \#MTU1437H }\end{array}$ & 141 \\
\hline 3 & $\begin{array}{l}\text { BERKIBARLAH PANJI ARROYAH } \\
\text { \#FokusHukumPenista } \\
\text { \#TangkapPenistaQuran } \\
\text { \#TolakPemimpinKafir https://t.co/DFwuN7UGRR }\end{array}$ & 81 \\
\hline 4 & $\begin{array}{l}\text { 5. Tudingan pun dimainkan dg dalih HTI anti } \\
\text { Pancasila, hanya karena kritis trhdp pemerintah } \\
\text { dan anti pemimpin kafir. \#BuletinAlIslam } 861\end{array}$ & 54 \\
\hline 5 & $\begin{array}{l}\text { \#FokusHukumPenista \#TangkapPenistaAlquran } \\
\text { \#TolakPemimpinKafir https://t.co/VoD3qyd6kp }\end{array}$ & 47 \\
\hline 6 & $\begin{array}{l}\text { Kibaran Bendera Nabi Semarakan Aksi } \\
\text { \#FokusHukumPenista \#TangkapPenistaquran } \\
\text { \#TolakPemimpinKafir https://t.co/lmVtqIUNtZ }\end{array}$ & 40 \\
\hline 7 & $\begin{array}{l}\text { Kibaran Bendera Nabi Semarakan Aksi } \\
\text { \#FokusHukumPenista \#TangkapPenistaquran } \\
\text { \#TolakPemimpinKafir https://t.co/DhOuwKAKh4 }\end{array}$ & 36 \\
\hline 8 & $\begin{array}{l}\text { Gubernur Kafir Boleh? Berbohong Atas Nama } \\
\text { Imam Al Mawardi dan Ibn Taimiyah } \\
\text { https://t.co/e3LOymc1RV }\end{array}$ & 36 \\
\hline 9 & $\begin{array}{l}\text { Demokrasi Ambil Alih Peran Tuhan. } \\
\text { Oleh Dr. Arim Nasim } \\
\text { \#FokusHukumPenista \#TangkapPenistaquran } \\
\text { \#TolakPemimpinKafir https://t.co/ZrfDKKIQzz }\end{array}$ & 34 \\
\hline 10 & $\begin{array}{l}\text { Kibaran Bendera Nabi Semarakan Aksi } \\
\text { \#FokusHukumPenista \#TangkapPenistaquran } \\
\text { \#TolakPemimpinKafir https://t.co/ojGkzS17Y1 }\end{array}$ & 31 \\
\hline
\end{tabular}

\section{Discussion}

From the result, we could see that both NU and HTI Twitter account uses negative sentiment word. However, NU uses less variation of negative sentiment word. The proof of this is that while the percentage is close $(5,7 \%(\mathrm{NU})$ compared to $6,5 \%(\mathrm{HTI})$ ) a number of negative sentiment words that are used in NU are significantly less from what is used in HTI account (246 word (NU) compared to 450 (HTI)).

We also could see that from the characteristic of the tweet, NU tend to post the tweet for religious speech, meanwhile, HTI mostly post tweets regarding their strong disagreement with the government decision, and some tweet contain blaming to the government. Overall HTI tweets persuade people who follow them to feel hatred toward government (See Table 5), and depending on the momentum, while Indonesian government tried to create rules to prevent terrorism in Indonesia, HTI tweets a tweet that creating an image that the government is the terrorist (See Table 6). 


\section{S. Conclusion}

Based on our research result, we could make several conclusion about the difference of language style used in social media between government-approved and radical moslem group in social media.

First, a government-approved moslem group mostly use their social media to give an online lecture about religion to their follower, meanwhile, radical Moslem group use their social media to persuade their follower to hate the government.

Second, because the tweet that is tweeted was taken directly from organization respective official account (NU and HTI), We could conclude that the data could represent both of religious group intention, based on data, NU plan is to give religious lecture toward people meanwhile HTI tried to persuade people by creating an image that government is a bad entity.

Therefore in the future, the method used in this paper could be used to support a government decision to differentiate between radical moslem group and normal Moslem group.

\section{REFERENCES}

[1] J. Klausen, "Tweeting the Jihad: Social Media Networks of Western Foreign Fighters in Syria and Iraq," Stud. Confl. Terror., vol. 38, no. 1, pp. 1-22, Jan. 2015.

[2] K. Makice, "Twitter API: Up and running: Learn how to build applications with the Twitter API," 2009.

[3] J. Roesslein, “Tweepy," Python Program. Lang. Modul., 2015.

[4] “Twitter Limit." [Online]. Available: https://dev.twitter.com/rest/reference/get/statuses/user_timeli ne. [Accessed: 14-Jan-2017].

[5] F. Tala, "A study of stemming effects on information retrieval in Bahasa Indonesia," Inst. Logic, Lang., 2003.

[6] I. Feinerer, "Introduction to the tm Package Text Mining in $\mathrm{R}, ”$, , edu. br/-kaestner/Min-eracao/RDataMining/tm, ..., 2017.

[7] S. Büttcher, C. Clarke, and G. Cormack, "Information retrieval: Implementing and evaluating search engines," 2016.

[8] R Development Core Team, "R Language Definition," Web, p. 62, 2011.

[9] D. Wahid and S. Azhari, "Peringkasan Sentimen Esktraktif di Twitter Menggunakan Hybrid TF-IDF dan Cosine Similarity," IJCCS (Indonesian J. Comput.

[10] B. Liu, M. Hu, and J. Cheng, "Opinion observer: analyzing and comparing opinions on the web," Proc. 14th Int. Conf., 2005.

[11] "TWEET USTADZ: Hormatilah Perempuan, Mereka Kaum Ibumu." [Online]. Available: http://www.poboonews.com/articlenew/48425593.html. [Accessed: 08-Jul-2017].

[12] "Kisah Syekh Abdul Qadir Al-Jilani Mimpi Bertemu Nabi -
Warta Islami Masa Kini | wartaislami.com.” [Online]. Available:

http://www.wartaislami.com/2017/06/kisah-syekh-abdul-qad ir-al-jilani-mimpi.html. [Accessed: 08-Jul-2017].

[13] "Wacanakan Asing Kelola Pulau, Luhut Dianggap Pelaku Makar Sesungguhnya | Media Informasi Rakyat." [Online]. Available:

http://www.postmetro.org/2017/01/wacanakan-asing-kelolapulau-luhut.html. [Accessed: 08-Jul-2017].

[14] "500 Investor Asing Ngemplang Pajak, Ini Membuktikan Bahwa... - Hizbut Tahrir Indonesia." [Online]. Available: https://hizbut-tahrir.or.id/2016/06/12/500-investor-asing-nge mplang-pajak-ini-membuktikan-bahwa/.

[Accessed: 08-Jul-2017].

[15] "Ancaman Masuknya Tenaga Kerja Asing bagi Keluarga dan Generasi - Hizbut Tahrir IndonesiaHizbut Tahrir Indonesia." [Online]. Available: https://hizbut-tahrir.or.id/2016/08/29/ancaman-masuknya-ten aga-kerja-asing-bagi-keluarga-dan-generasi/. [Accessed: 08-Jul-2017].

[16] "Talkshow Interaktif HTI Bandar Lampung: 'Revisi UU Terorisme Membidik Islam?' - Hizbut Tahrir Indonesia." [Online]. Available: https://hizbut-tahrir.or.id/2016/04/01/talkshow-interaktif-htibandar-lampung-revisi-uu-terorisme-membidik-islam/. [Accessed: 08-Jul-2017].

[17] "Ketua Pansus RUU Terorisme : Yang Tidak Sejalan Dengan Barat Dicap Teroris - Hizbut Tahrir Indonesia." [Online]. Available:

https://hizbut-tahrir.or.id/2016/07/27/ketua-pansus-ruu-terori sme-yang-tidak-sejalan-dengan-barat-dicap-teroris/.

[Accessed: 08-Jul-2017].

[18] "Penerapan Khilafah di Bumi Nusantara, Utopia Atau Keniscayaan?" [Online]. Available: http://www.harianjawabarat.com/2017/05/penerapan-khilafa h-di-bumi-nusantara-utopia-atau-keniscayaan/. [Accessed: 08-Jul-2017]. 